\title{
Cytomegalovirus-mononucleosis-induced thyroiditis in an immunocompetent patient
}

\author{
V Larouche ${ }^{1}$ and M Tamilia ${ }^{2}$ \\ 1Resident, Adult Endocrinology and Metabolism Training Program, McGill University, Montréal, Québec, Canada and \\ 2Division of Endocrinology, Jewish General Hospital, Montréal, Québec, Canada
}

Correspondence should be addressed to $\mathrm{V}$ Larouche Email

Vincent.larouche@mail. mcgill.ca

\section{Summary}

Enteroviruses, including coxsackieviruses and Echovirus, are well known pathogens responsible for the development of thyroiditis. We describe the case of a 49-year-old woman with no personal or family history of thyroid disease who presented to the emergency room with a two-week history of daily fevers up to $39^{\circ} \mathrm{C}$, a sore throat, occasional palpitations and diaphoresis, decreased appetite and an unintentional $10 \mathrm{~kg}$ weight loss over the same time course Physical examination revealed mild tachycardia, an intention tremor and a normal-sized, nontender thyroid gland without palpable nodules. The remainder of the physical examination was unremarkable and without stigmata of Graves' disease. Her initial blood tests revealed overt thyrotoxicosis, elevated liver enzymes, an elevated C-reactive protein, a negative monospot and a positive CMV IgM antibody. Thyroid sonography revealed areas of hypoechogenicity and relatively low vascularity. Fine-needle biopsy showed a lymphocytic infiltrate. The patient was treated symptomatically with propranolol. On follow-up, the patient became euthyroid, and her liver enzymes normalised. Previous cases of CMV-induced thyroiditis occurred in immunosuppressed patients. This is the first reported case of a CMV-mononucleosisinduced thyroiditis in an immunocompetent adult patient and serves as a reminder that viral illnesses are a common cause of thyroiditis with abnormal liver enzymes.

\section{Learning points:}

- The differential diagnosis of thyrotoxicosis with abnormal liver enzymes includes severe hyperthyroidism and thyroid storm caused by Graves' disease as well as the thyrotoxic phase of a thyroiditis, usually caused by a virus such as coxsackievirus or, in this case, cytomegalovirus.

- Cytomegalovirus appears to be a recently recognized causal agent for thyroiditis, both in immunosuppressed and immunocompetent patients.

- Careful follow-up of thyroid function tests in patients with thyroiditis allows clinicians to determine if patients' thyroid hormone secretion normalizes or if they remain hypothyroid.

\section{Background}

Subacute thyroiditis is characterized by an initial phase of thyrotoxicosis with neck pain, a tender diffuse goiter and elevated thyroxine (T4) and/or triiodothyronine (T3). The classic pattern of changes in thyroid function in patients with thyroiditis is thyrotoxicosis, followed by hypothyroidism, and then recovery within 8-12 weeks.
During the thyrotoxic phase, thyroid hormone synthesis and thyroid radioiodine uptake are low, in contrast to Graves' disease in which they are elevated. Therefore, the thyrotoxicosis lasts only until the stores of T4 and T3 are depleted, which corresponds usually to a two- to six-week period. Hypothyroidism is also 
usually transient, but can occasionally be permanent. Lymphocytic thyroiditis is painless and often referred to as silent thyroiditis.

A viral infection or post-viral inflammatory process is thought to cause thyroiditis, because many patients have a history of an upper respiratory tract infection preceding the onset, and clusters of cases have occurred in association with epidemics of coxsackievirus or other enteroviruses (1).

To our knowledge, this case is the first report of a cytomegalovirus-mononucleosis-induced thyroiditis in an immunocompetent adult patient, as other cases were reported in an immunosuppressed adult and in an infant.

\section{Case presentation}

A 49-year-old woman with no personal or family history of thyroid disease, primary or secondary immune deficiency and who takes no medication including any immunosuppressant, presented to the emergency room of our hospital with a two-week history of daily fevers up to $39^{\circ} \mathrm{C}$, a sore throat, occasional palpitations and diaphoresis, decreased appetite and an unintentional $10 \mathrm{~kg}$ weight loss over the same time course. She denied ocular symptoms, compressive symptoms, anterior neck pain or other symptoms of thyrotoxicosis.

Physical examination revealed mild tachycardia (HR $105 \mathrm{bpm}$, regular) with normal vital signs otherwise, an intention tremor and a normal-sized, nontender thyroid gland without palpable nodules. The patient had no thyroid stare or lid lag. The remainder of the physical examination was unremarkable and without stigmata of Graves' disease, such as ophthalmopathy or dermopathy.

Her initial blood test revealed overt thyrotoxicosis (TSH: $0.06 \mathrm{U} / \mathrm{L}$ (normal: $0.40-4.59 \mathrm{U} / \mathrm{L}$ )), free T4: $48.9 \mathrm{pmol} / \mathrm{L}$ (normal: $9.0-26.0 \mathrm{pmol} / \mathrm{L}$ ), elevated transaminase levels (ALT: $261 \mathrm{U} / \mathrm{L}$ (normal: 5.0-40.0 U/L)), AST: 203U/L (normal: 15.0-55.0U/L), an elevated
C-reactive protein at $23.3 \mathrm{mg} / \mathrm{L}$ (normal: $0-10.0 \mathrm{mg} / \mathrm{L}$ ) and a negative monospot but a positive CMV IgM antibody (c.f. Table 1). Of note, thyroglobulin, thyroglobulin antibodies and anti-TPO antibodies were not ordered. The infectious disease service diagnosed acute active cytomegalovirus mononucleosis and treated her conservatively.

The next morning, she was seen in Endocrinology Clinic and given the clinical suspicion of thyroiditis; she was treated symptomatically.

\section{Investigation}

Seven days after her initial presentation, a 24-h radioactive iodine uptake disclosed a $24 \%$ uptake of I-131 at $24 \mathrm{~h}$, the normal range being $8-28 \%$ at our institution (Fig. 1). The uptake was uniform in bilateral thyroid lobes with a photopenic region in the right superior lobe. Although we recognize it is not typical of the thyrotoxic phase of a painless thyroiditis, given the clinical context of acute CMV mononucleosis concurring with thyrotoxicosis, we interpreted the normal range I-131 uptake on thyroid scan as a resolving thyroiditis as it was probably performed in the recovery or transition phase.

TSH receptor antibody titer was weakly positive. We believe that the mildly positive TSH receptor antibodies are suggestive of a sensitization process during the course of the thyroiditis, rather than Graves' disease. This is supported by the fact that the antibody titer as well as sonographic features normalized over time during follow-up .

Three weeks after her initial ER visit, a thyroid sonography revealed areas of hypoechogenicity and relatively low vascularity diffusely in the thyroid gland. Fine-needle aspiration biopsy of the area in the right superior thyroid lobe corresponding to a photopenic area on the uptake scan was performed the same day. No nodule or cyst was seen on the thyroid ultrasound, suggesting that the photopenic region may have been due to inflammation. Of note, the ultrasound was not

Table 1 Biochemical investigation of the patient.

\begin{tabular}{|c|c|c|c|}
\hline Test & Day 1 & Day 7 & Day 10 \\
\hline TSH & 0.06 & 0.31 & 0.22 \\
\hline Free T4 & 48.9 & 23.3 & 22.8 \\
\hline Free T3 & & 6.2 & \\
\hline TRAb & & 3.0 & \\
\hline ALT & 261 & 134 & 95 \\
\hline AST & 203 & & 55 \\
\hline CRP & 23.3 & & \\
\hline CMV IgM & Positive & & \\
\hline Monospot & Negative & & \\
\hline
\end{tabular}

\begin{tabular}{ccc}
\hline Day 37 & & Day $\mathbf{4 2 7}$ \\
\cline { 1 - 1 } 1.19 & & 2.71 \\
16.2 & & 15.8 \\
5.1 & & 4.9 \\
& & 0.8 \\
21 & & 21 \\
& 2.5 \\
& \\
& \\
\hline
\end{tabular}

\begin{tabular}{c}
\hline Normal range \\
\hline $0.40-4.59 \mathrm{U} / \mathrm{L}$ \\
$9.0-26.0 \mathrm{pmol} / \mathrm{L}$ \\
$2.8-7.1 \mathrm{pmol} / \mathrm{L}$ \\
$0-1.2 \mathrm{U} / \mathrm{L}$ \\
$5.0-40.0 \mathrm{U} / \mathrm{L}$ \\
$15.0-55.0 \mathrm{U} / \mathrm{L}$ \\
$0-10.0 \mathrm{mg} / \mathrm{L}$ \\
Negative \\
Negative
\end{tabular}

http://www.edmcasereports.com 


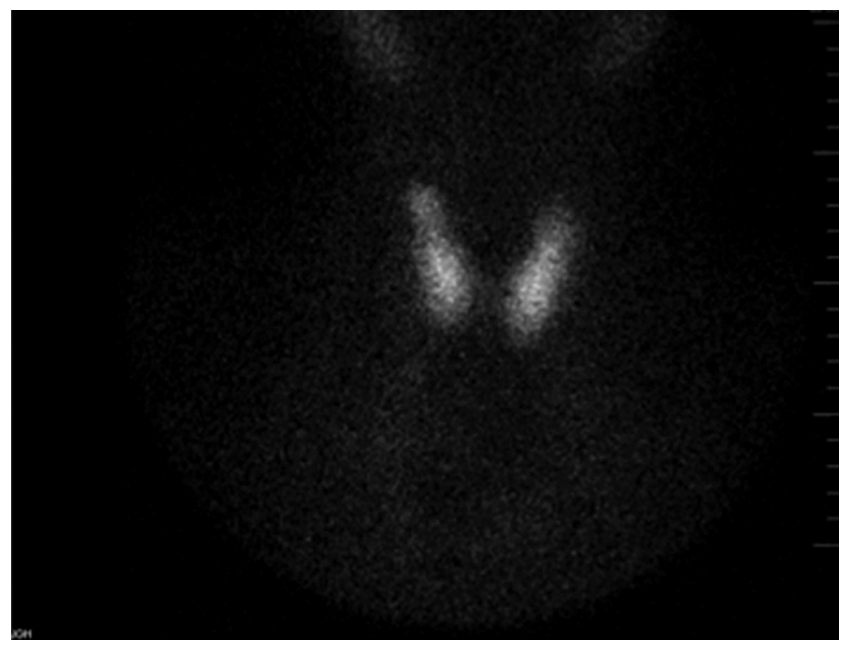

Figure 1

Radioactive iodine uptake scan.

repeated during follow-up. Pathology report described a small amount of colloid and few strips of small follicular cells. The pathologist interpreted the FNA biopsy result as nondiagnostic and the CMV immunostain was negative, which may have been secondary to the paucity of cells in the sample.

Overall, given the clinical picture, we hypothesized that this immunocompetent 49-year-old woman had developed thyroiditis as a consequence of her CMV mononucleosis. Other diagnostic possibilities include incipient Graves' disease or a viral-induced autoimmune thyroiditis. Given this differential diagnosis, we ensured the patient is followed with regular thyroid function tests to monitor for development of hyperthyroidism.

\section{Treatment}

For her adrenergic symptoms, the patient was started on propranolol $40 \mathrm{mg}$ po bid on her first visit to the Endocrinology clinic. However, the patient discontinued propranolol on her own as she felt it did not improve her symptoms. Due to the absence of thyroid pain (spontaneous or upon palpation), she was given neither NSAIDs nor prednisone. Given that thyroiditis was our most likely diagnosis, we did not start the patient on any anti-thyroid drug such as methimazole or propylthiouracil.

\section{Outcome and follow-up}

During the follow-up period of sixteen months, the patient had no recurrence of symptoms of thyrotoxicosis.
Similarly, she always remained biochemically euthyroid, her TSH receptor antibodies and her liver enzymes normalised during follow-up.

\section{Discussion}

In summary, our patient is an immunocompetent 49-year-old woman who developed thyroiditis following an episode of cytomegalovirus-induced mononucleosis, explaining her presenting symptoms and clinical picture.

A MEDLINE search from 1950 to the present using the terms 'cytomegalovirus', 'thyroiditis', 'hyperthyroidism', 'thyrotoxicosis, 'CMV' and 'mononucleosis' found two similar case reports.

André and coworkers (2) reported a 47-year-old man with psoriatic arthritis treated with infliximab who presented with daily fevers. Apart from fever, his result was unremarkable and his thyroid was nontender. His thyroid function tests showed overt thyrotoxicosis, a high CRP, negative TSH receptor antibodies, but a high CMV PCR. His thyroid uptake scan showed low uptake and ultrasound showed increased gland volume with hypoechoic signal. The patient's symptoms, thyroid function tests and CMV PCR normalized with holding infliximab.

Al Maawali and coworkers (3) described an 18-month old infant girl who developed a painful subacute thyroiditis secondary to CMV mononucleosis. She had high fevers and a mildly painful right neck mass. Her thyroid function tests were normal, but she had a high WBC count, high CRP and ESR and CMV IgM count. Her thyroid ultrasound revealed a diffuse hypoechogenic enlargement of her right lobe without abscess formation. Her thyroglobulin titer was elevated. FNA biopsy was negative for Gram stain and culture. TSH receptor antibodies and thyroid uptake scan were not performed in this infant. She was initially started on amoxicillin-clavulanate, but it was later stopped, and she was managed symptomatically.

Our patient had similar symptoms, physical examination findings (including a painless thyroid) and laboratory findings as in case by André and coworkers. However, as opposed to that case, our patient was immunocompetent, her thyroid uptake was within normal range at seven days and her TSH receptor antibodies were transiently mildly positive.

When compared to case by $\mathrm{Al}$ Maawali and coworkers, our patient did not have a painful thyroid, but her laboratory and ultrasonographic features were similar. As TSH receptor antibodies were not drawn and a 
thyroid uptake scan was not performed for this infant girl, comparison between both cases is limited.

In a review by Desailloud and coworkers (4), one article revealed positive cultures for mumps virus in biopsies of two patients out of a cohort of 11 patients with subacute thyroiditis and mumps during an epidemic (5). With regards to serological evidence of acute viral infection in patients with subacute thyroiditis, a study of 58 patients with subacute thyroiditis, who had no clinical evidence of viral disease, viral antibody titers of 32 patients demonstrated increases at least fourfold. These included antibodies to mumps virus, but also coxsackie, adenovirus and influenzae. Coxsackie viral antibodies were the most commonly found, and the changes in their titers most closely approximated the course of the disease (6).

In conclusion, there seems to be an association between cytomegalovirus as a causal agent for painless thyroiditis as well as painful thyroiditis. Laboratory and imaging findings may differ between cases. The age and integrity of the patient's immune system may explain these clinical differences. Although direct thyroid invasion or serological evidence of acute infection have been reported in patients with thyroiditis and other viruses, more extensive research is needed to elucidate the link between cytomegalovirus and thyroiditis to help guide clinicians in managing such cases.

\section{Declaration of interest}

The authors declare that there is no conflict of interest that could be perceived as prejudicing the impartiality of the research reported.

\section{Funding}

The research did not receive any specific grant from any funding agency in the public, commercial or not-for-profit sector.

\section{Patient consent}

The patient provided written consent for the publication of this article.

\section{Author contribution statement}

Dr M Tamilia is the Endocrinologist who was on service and initially saw the patient in clinic the day following her ER presentation. He also performed an ultrasound and a fine-needle aspiration biopsy on the patient's thyroid. Dr V Larouche is the Endocrinology resident who was on service, saw the patient with Dr M Tamilia and followed up her case in clinic in the weeks following her initial presentation.

\section{References}

1 Ross DS, Burch HB, Cooper DS, Greenlee MC, Laurberg P, Maia AL, Rivkees SA, Samuels M, Sosa JA, Stan MN, et al. 2016 American Thyroid Association Guidelines for diagnosis and management of hyperthyroidism and other causes of thyrotoxicosis. Thyroid 26 1343-1421. (https://doi.org/10.1089/thy.2016.0229)

2 André R, Opris A, Costantino F, Hayem G \& Breban M 2016 Cytomegalovirus subacute thyroiditis in a patient treated by infliximab for psoriatic arthritis. Joint Bone Spine 83 109-110. (https://doi.org/10.1016/j.jbspin.2015.06.001)

3 Al-Maawali A, Al Yaarubi S \& Al Futaisi A 2008 An infant with cytomegalovirus-induced subacute thyroiditis. Journal of Pediatric Endocrinology and Metabolism 21 191-193.

4 Desailloud R \& Hober D 2009 Viruses and thyroiditis: an update. Journal of Virology 12 1-14. (https://doi.org/10.1186/1743-422X-6-5)

5 Eylan E, Zmucky R \& Sheba C 1957 Mumps virus and subacute thyroiditis. Evidence of a causal association. Lancet 272 1062-1067. (https://doi.org/10.1016/S0140-6736(57)91438-1)

6 Volpe R, Row VV \& Ezrin C 1967 Circulating viral and thyroid antibodies in subacute thyroiditis. Journal of Clinical Endocrinology and Metabolism 27 1275-1284. (https://doi.org/10.1210/jcem-27-9-1275)

Received in final form 220ctober2017 Accepted 2November2017 